\title{
Bridging Socially Enhanced Virtual Communities
}

\author{
Venugopal Rao ${ }^{1}$, R.V.Krishnaiah ${ }^{2}$ \\ ${ }^{I}$ (Department of CSE, DRK Institute of Science \& Technology, India) \\ Principal \\ ${ }^{2}$ (Department of CSE, DRK Institute of Science \& Technology, India)
}

\begin{abstract}
As technologies required for collaborative computing grow, there are business collaborations among companies carrying out related business. The communication and interactions across the companies has become a common phenomenon. In order to create and sustain this kind of business collaborations, specialized methods or techniques are required. In this paper we propose concept of bridging socially enhanced virtual communities. It is achieved by discovering present relevant businesses and prospective business alliances by developing a semi-automated approach. The architecture of the proposed system is compromising of human activities and also automated activities and it is essentially a service oriented system. For the purpose of bringing the previously separated groups and also finding new relevant groups we introduced broker discovery concept that makes use of techniques and metrics pertaining to interactive mining. The empirical results revealed that the proposed system is effective when tested with test bed which is based on distributed technology such as web services.
\end{abstract}

Index Terms: Social interactions, interaction mining, broker discovery, business collaborations

\section{Introduction}

The advancements in distributed computing and infrastructure pertaining Information and Communication Technology has paved the way for potential collaborations among companies doing relevant business [1]. Especially the technologies like web services that work on XML (eXtensisble Markup Language) has practically made it possible for companies to have realistic alliances virtually. This has led to the formation of chain of companies or group of companies that involve in customer centric business processes. For instance, the banking sector is fully making use of the distributed technologies like web services to leverage and customize its consumer centric policies and making banking services available to its customers in many ways. However, there are some obstacles to establish such collaborations among companies. They include resources and services are to be discovered dynamically and context aware and flexible interactions among people of different companies and also different departments of same company. In social networking interactions are from various individuals or organizations that have influenced the emergence of many techniques pertaining to interaction mining and knowledge discovery. However, the social networking structures have relatively small information paths [2]. As the social networks are organizational units, the collaborations are restricted. In this paper we propose a social networking principle in order to bridge disjoint collaborative networks. The contributions of our research include introduction of brokers to connect sub groups; metrics to support discovery and selection of brokers; BQDL (Broker Query and Discovery Language). The rest of the paper describes related work and the concepts of this paper in theory and practical.

\section{Related Work}

Service oriented systems are becoming stable and practical as there are standards established that are followed globally [3]. There is an important aspect to be observed in the service oriented systems. Though the technologies like web services support heterogeneous systems to be connected seamlessly, the human intervention is required some where in the business process. Therefore it is essential to consider human-based process as part of the whole service based systems. Hence, in our system we mix the service based systems based on SOA and also social principles. In order to support flexible service oriented systems among diverse organizations, we adopt HPS (Human Provided Services) which is proved to be suitable [4]. Simple interaction models are supported by existing platforms while social network principles can support advanced techniques like formation and adaptive coordination.

Burt [5] proposed theory known as "structural holes". It depends on the hypothesis that individuals who serve as intermediaries can be benefited. In our work, we use strategic formation of communities and social networks as described in [6]. In [7] broker incentive techniques are used which are based on game theoretic analysis. The proposed system is based on interaction mining. It also makes use of metrics that help in discovering brokers dynamically. The amount of information available on human interactions in social networking has closed a loop [2] that allows forming models that can help in the design of applications such as 
crowd sourcing techniques [8]. In [9], [10], many trust models have been described. However, we make use of social trust models described in [11], [12], [13]. For improving queries on social networking information, we proposed BQDL which is intuitive in nature. SPARQL [14] is a generic query language while BQDL is not and allows specific discovery of actors in the social landscape.

\section{Virtual Communities}

An emerging virtual community in the social and collaborative landscape is Professional Virtual Community (PVC). It has actors who interact with others through a well established ICT. In order to realize this service oriented systems are used. The emergence of distributed computing technologies has paved way to have web-enabled PVCs.

\subsection{Collaboration Brokering in PVC}

PVC allows experts from various organizations to have collaborations through social networking or other customized applications. The experts are considered actors while the actions they perform are known as activities. They use SOA based technologies in order to form a virtual community that can have flexible interactions. HPS framework [4] is such technology that supports human interactions and participation in service oriented system. The members of PVC can benefit from other experts as they can share knowledge, clarify doubts of others and also help each other in their professions. The PVC network comprised sub domains that have groups of experts.

Broker concept helps in connecting different sub groups in the virtual community. A person from a domain may be acting as broker who can help connect a known person with other domain. Inter - domain relations are improved by using broker concept. It also leverages finding new groups that can join the PVC thus supporting the formation of expert groups. The relationships among the actors can be converted into FOAF relations. This is because people in general trust known people when compared with unknown people. Therefore the broker concept has its impact on the scenarios such as formation of expert groups; controlling interactions and delegations; establishment of new social relations.

\section{Socially Enhanced Virtual Communities}

This section focuses on flexible service oriented collaborations, trust relationships and broker behavioral patterns.

\subsection{Service Oriented Collaboration}

SOA is basis for service oriented collaborations. While this is the fact, the HPS can enhance collaborations containing software services and also human activities together. Thus it is capable of forming flexible service oriented collaboration. In order to achieve this steps followed are publish, search and interact. Publish does mean that people can develop HPS and publish them to make it available to public. Search allows people to search for services suitable to their needs and Interact is for providing personalized interaction environments that are intuitive and usable.

\subsection{Trust Evolution in PVC}

The trust being described here is not related to security but social in nature. It depends on the collaborations and the behavior in the previous such collaborations as per the needs and changing interests of individuals [11], [12]. As people in collaborative network involve in business and the trust is required in order to ensure that collaborations are productive and long lasting. The trust model proposed by us is influenced by [15], [10] and [12]. Interaction loges are used to derive metrics that help in finding facts and relationships among actors in right perspective. The proposed system is also based on HPS concept. One example for metric is the success rate of delegated tasks between members. These metrics help in finding trustworthy collaborations. The interaction metrics such as responsiveness, success rate are used in our work to promote trust relationships. Further the trust is based on the context and scope.

\section{The BQDL And Its Specifications}

BQDL is a language that has syntax similar to SQL. It is meant for querying data from the interactions of collaborative and socially enhanced virtual communities such as PVC. The important terms or keywords of this language are described in table 1 . 


\begin{tabular}{|l|l|}
\hline ELEMENT & DESCRIPTION \\
\hline[] & $\begin{array}{l}\text { Used to make an expression to represent one, one to m }[1 . . \mathrm{m}] \text { and one to many }\left[1 . .^{*}\right] \\
\text { relationships. }\end{array}$ \\
\hline$<$ all> & Retains all nodes that satisfy given criteria. \\
\hline as & Used to create an alias \\
\hline satisfy & Requires that given criteria should be satisfied. \\
\hline
\end{tabular}

Table 1: Shows elements of BQDL

When there are many communities and finding required information among the communities is possible with BQDL language expressions. The figure 1 shows the BQDL script that is used to obtain suitable broker to connect two predefined communities.

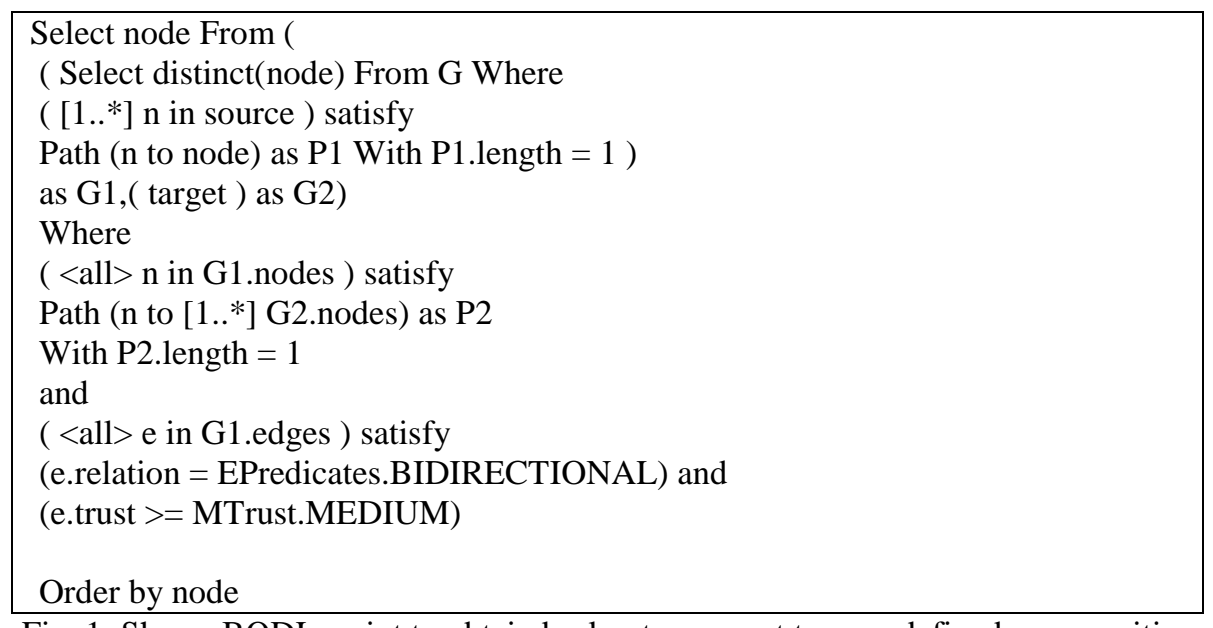

Fig. 1: Shows BQDL script to obtain broker to connect two predefined communities

As can be seen in the above figure, the BQDL is used to find out broker suitable to connect two predefined communities. The query also makes use of trust values. The predicate is used to obtain only required communities. The above query can also be given to real world system with some form of GUI or web based interface to make it user friendly.

\section{Implementation}

As part of the proposed system that leverages the social enhanced virtual communities, BQDL is instrumental in achieving this. The implementation is making use of web services that help integrate even heterogeneous applications that is the need of the hour in the real world. A broker discovery application has been built in order to test the concepts and BQDL proposed in this paper.

\subsection{Broker Discovery Application}

The web based application which has been implemented can be considered a broker discovery tool that makes use of a query language such as BQDL. It internally is based on the concept of FOAF that is the basis for brokers in order to connect previously disconnected communities and also add new communities into the existing virtual communities' network which is based on SOA technologies. The result of BQDL query is a visualization of graph comprising brokers that can be used to connect disjoint social communities.

\subsection{TESTBED}

Genesis2 framework is used in Linux operating environment. The SOA based PVCs established by Genesis2 web services that has capabilities to simulate communities are used. Minimum spanning trees algorithm is used in the application of broker discovery. Amazon EC2 cloud has been used for experiments.

\subsection{PERFORMANCE EVALUATION OF BQDL}

The BQDL language has been tested in the given test bed configuration. The testing has been done under various characteristics such as changing nodes and groups. The libraries pertaining to graph are implemented in C\# which is used in BQDL. The queries made by changing number of tags, nodes, communities or groups revealed that the BQDL processing time is based on the experiment parameters considered. The $\mathrm{BQDL}$ processing time for various experiments is shown in the table given below. 


\begin{tabular}{|l|l|l|l|l|l|}
\hline EXPERIMENT & \#REQ & MIN & AVG & MAX & TOAL \\
\hline $1(\mathrm{RP}=10)$ & 50 & 3167 & 9000 & 10349 & 52543 \\
& 100 & 1650 & 9369 & 10345 & 101240 \\
& 200 & 1800 & 9201 & 10734 & 190640 \\
\hline $1(\mathrm{RP}=50)$ & 50 & 1606 & 15955 & 29952 & 50763 \\
& 100 & 1482 & 27440 & 48562 & 98685 \\
& 200 & 1639 & 36313 & 47687 & 188573 \\
\hline $1(\mathrm{RP}=100)$ & 50 & 1606 & 15955 & 29952 & 50762 \\
& 100 & 1544 & 28560 & 57501 & 105331 \\
& 200 & 1591 & 55185 & 100370 & 202394 \\
\hline
\end{tabular}

Table 2: Shows Processing Time of BQDL

As can be seen in table 2, the results reveal that the processing time gets increased when number of requests is increased. RP represents concurrent requests. The influence of RP is less on the results while the number of requests has impact on the processing time of BQDL.

\section{Conclusion}

This paper proposed the brokers concept in social enhanced systems that are based on SOA. The concept is taken from the structural holes which is part of social sciences. Generally service oriented systems comprise of services. However, human modeled system integrating with SOA is required as the human element will act as broker in the system used to connect disjoint communities. Interaction mining techniques are used to achieve this. The BQDL query language has been proposed that is meant for making user friendly queries on interactions of virtual communities and also discovering brokers. The present system also uses trust model that helps in increasing the reliability of the system in social aspect. The system can be enhanced in future by implementing link based reputation ranking algorithm for brokers.

\section{References}

[1] L. M. Camarinha-Matos and H. Afsarmanesh.Collaborative networks. In PROLAMAT, pages 26-40,2006.

[2] J. Kleinberg. The convergence of social and technological networks. Commun. ACM, 51(11):66-72,2008.

[3] M. Ford et al. Web Services Human Task (WS-HumanTask), Version 1.0., 2007.

[4] D. Schall, H.-L. Truong, and S. Dustdar. Unifying human and software services in web-scale collaborations. Internet Comp., 12(3):62-68, 2008.

[5] R. S. Burt. Structural holes and good ideas. American Journal of Sociology, 110(2):349-399, Sept. 2004.

[6] W. Tsai. Social capital, strategic relatedness, and the formation of intra-organizational strategic linkages. Strategic Management Journal, (21):925-939, 2000.

[7] J. Kleinberg, S. Suri, E. Tardos, and T. Wexler. Strategic network formation with structural holes.ACM Conference on Electronic Commerce, 7(3):1-4, 2008.

[8] D. Brabham. Crowd sourcing as a model for problem solving: an introduction and cases. Convergence, 14(1):75, 2008.

[9] D. Artz and Y. Gil. A survey of trust in computer science and the semantic web. J. Web Sem., 5(2):58-71, 2007.

[10] L. Mui, M. Mohtashemi, and A. Halberstadt. A computational model of trust and reputation for e-businesses. In HICSS, page 188, 2002.

[11] J. Golbeck. Trust and nuanced profile similarity in online social networks. TWEB, 3(4), 2009.

[12] F. Skopik, D. Schall, and S. Dustdar. Modeling and mining of dynamic trust in complex service-oriented systems. Information Systems, pages 735-757, 2010.

[13] C.-N. Ziegler and J. Golbeck. Investigating interactions of trust and interest similarity. Decision Support Systems, 43(2):460-475, 2007.

[14] W3C. Sparql query language for rdf, 2008. Online: http://www.w3.org/TR/rdf-sparql-query/.

[15] T. Grandison and M. Sloman. A survey of trust in internet applications. IEEE Communications Surveys and Tutorials, 3(4), 2000.

\section{AUTHORS}

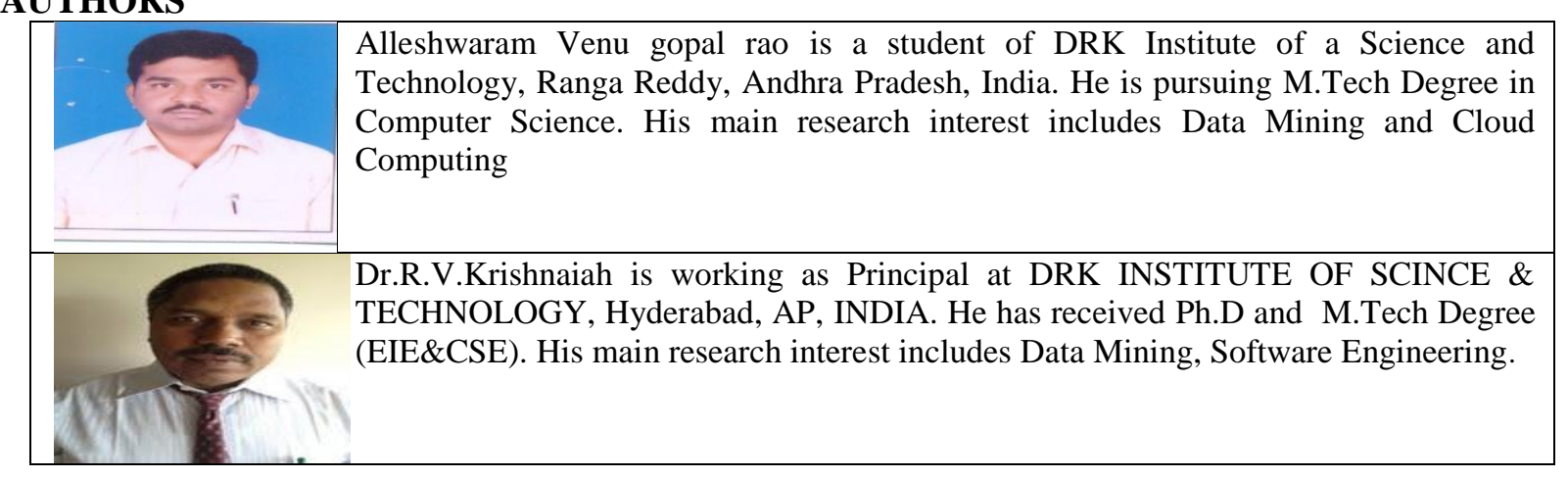

